\title{
ADVANCES IN THE UNDERSTANDING AND OPERATIONS OF SUPERCONDUCTING COLLIDERS
}

\author{
G. Annala, P. Bauer ${ }^{\dagger}$, L. Bottura ${ }^{\#}$, M.A. Martens, N. Sammut ${ }^{\#}$, G. Velev, V. Shiltsev \\ FNAL, Batavia, IL, USA, "CERN, Geneva, Switzerland
}

\begin{abstract}
Chromaticity drift during injection is a well-known phenomenon in superconducting colliders, such as the Tevatron, HERA and RHIC. Imperfect compensation of the drift effects can contribute to beam loss and emittance growth. It is caused by the drift of the sextupole component in the dipole magnets due to current redistribution in its superconducting coils. Recently extensive studies of chromaticity drift were conducted at the Tevatron, aiming at the improvement of the luminosity performance in the ongoing run II. These studies included not only beam experiments, but also extensive off-line magnetic measurements on spare Tevatron dipoles.

Less known, until recently, is that chromaticity drift is often accompanied by tune and coupling drift. This was recently discovered in the Tevatron. We believe that these effects are the product of systematic beam offset in conjunction with the sextupole drifts (and their compensation in the chromaticity correctors).

These discoveries are most relevant to the upcoming LHC, where the drift effects will have even more dramatic consequences given the high beam current. It is therefore not a surprise that CERN has been the source of major advances in the understanding of dynamic effects during the LHC superconducting magnet development.

The following will briefly review the CERN results as well as those of the recent Fermilab studies. A new result, which will be presented here also, is related to fast drifts occurring in the first few seconds of the injection plateau. Again, these fast drifts were observed first in the Tevatron and efforts are underway to explain them. Finally this paper will attempt to derive the implications of these drift effects on LHC commissioning and operation.
\end{abstract}

\section{DRIFT \& SNAPBACK}

Almost twenty years passed since the seminal paper by Finley et al. at the PAC of 1987 ([1]), which reported the discovery of chromaticity drift in the Tevatron as well as discussed drift of the sextupole $\left(b_{2}{ }^{*}\right)$ component in the superconducting dipole magnets as possible cause. Soon it

\section{†pbauer@fnal.gov}

\footnotetext{
* In an accelerator dipole magnet the 2-dimensional magnetic field is expanded in complex harmonics, where $b_{n}$ and $a_{\mathrm{n}}$ are the normal and skew multipole coefficients, respectively, and $\mathrm{R}_{\text {ref }}$ is the reference radius (17 mm for the LHC and 1 inch for the Tevatron). Multipoles (or "field errors”) are expressed in units of $10^{-4}$ with respect to the main dipolar component $\mathrm{B}_{0}$.
}

was also discovered that the drift in the $b_{2}$ component is followed by a rapid snap-back at the start of the ramp from the injection plateau [2]. Fig. 1 shows a typical example of $b_{2}$ drift and snapback in a Tevatron dipole magnet. The insert in Fig. 1 shows how the $b_{2}$ departs from the hysteretic baseline during the dwell at the injection porch. At present, based on magnetic measurements of LHC series dipoles, a double exponential dependence on time appears to be appropriate to describe the drift (the same applies to RHIC dipoles). A logarithmic function was chosen to describe the behavior of the Tevatron dipoles (Eq. 1).

$b_{n}^{d r i f t}(t)=b_{n, 0}+m \ln \left(\frac{t+t_{0}}{t_{0}}\right)$

The offset factor $b_{n, 0}$ allows for the inclusion of a "fastdrift" term, as recently discovered in the Tevatron. This will be discussed in further detail below and in other, related contributions to these proceedings $[21,22]$.

Decay and snapback, referred to as dynamic effects in the superconducting magnet community, also appear in other, allowed (as well as un-allowed!) magnetic multipoles. In the allowed multipoles the drift always reduces the magnetization contribution, because it is the result of a superconductor de-magnetization. As shown in Fig. 1, for instance, the $b_{2}$ increases during the drift. In the case of the non-allowed multipoles no such rule exists. In the Tevatron dipoles, for example, small skew quadrupole drifts toward larger and smaller values have been observed [20]. In some instances the drift even reversed sign. The skew quadrupole drift is the result of the same process that gives rise to sextupole drift, except that, in a dipole for example, it is the difference between the contributions to the effect emanating from the two poles.

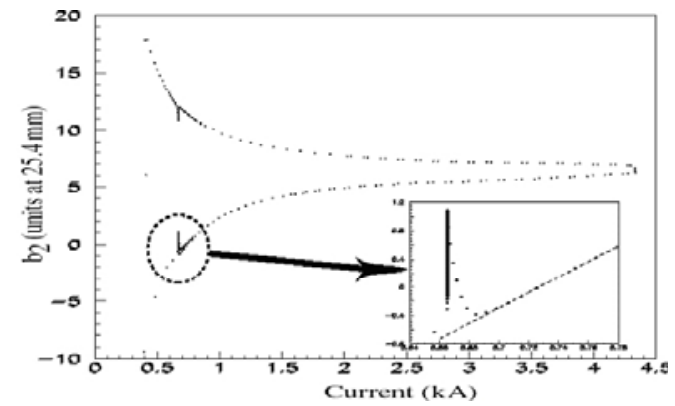

Figure 1: Examples of sextupole drift and snapback (see insert) in a Tevatron dipole magnet. 


\section{DYNAMIC EFFECT MODEL}

First it was thought that the dynamic effects are the result of flux creep in the superconductor. This effect, however, had to be relegated to be of lesser importance, when Kuchnir's measurements revealed flux creep rates in Tevatron magnet cables ([4]) that were ten times smaller than those observed in magnets. The more recent model, first proposed by Krempasky [5], Verweij [6], Bottura [8] and Wolf [9] on the basis of an idea by R. Stiening [10], postulates that the effect is caused by the re-distribution of currents between strands in the cables making up the coils in conjunction with superconductor demagnetization. [6] refers to these currents as BICC, or boundary induced coupling currents, since they originate in the magnet ends or splices. Haverkamp recently demonstrated this experimentally, measuring the demagnetization of a single strand of the LHC type, around which he wound six copper strands that were injected with currents that simulated the current redistribution in a cable [11].

Current redistribution occurs between the strands in the cables through the complex network of contact resistances (and through the splices). The characteristic time constant of this process, which can be thousands of secs with the currents running mostly within superconductor, is consistent with the time constant of the $b_{2}$ drift in the magnets [6]. As currents redistribute, the superconducting filaments within the strands see minute changes of the local magnetic field. If these local changes in magnetic field are not overwhelmed by the change of field caused by a change in transport current in the magnet, such as during ramping, the superconductor magnetization will also change as a result of it. The current redistribution model also naturally explains the strong correlation found in the dynamic effects of all allowed multipoles [7]. It also explains the so-called periodic pattern, a modulation of the allowed magnetic multipoles (incl. the main component), at the ten units level. The pattern period is approximately the twist pitch of the (inner layer) cable and its amplitude evolves in time with a similar timeconstant as the $b_{2}$ decay. In fact, we know today that the pattern is the "direct" signature of a current imbalance in the cable on the bore field.

When a superconductor, such as the microscopic filaments in the strands of the cables in the Tevatron or LHC magnets, is exposed to an external magnetic field, super-currents are generated that partially shield the inside of the filaments from that field. As the external field is decreased the superconductor traps field inside, again generating a magnetic moment that is opposed to the external field change. This magnetization produces most of the low field hysteresis in the superconducting magnets. When the field is ramped up, the strand magnetization evolves along the hysteretic loop. A sudden reversal to a decreasing field produces a very strong change of the superconductor magnetization, as one flips to the "other" side of the hysteretic loop. This strong change is the result of an instantaneous excitation of a new shielding current with opposite polarity. The shielding current reversal has a much larger effect on the superconductor magnetization than a small change of field along the hysteretic loop would have produced. This is the reason for the nonlinear magnetization response of the superconductor.

When the current ramp is interrupted to initiate beam injection at the injection porch, the ever-present current re-distribution will produce a local magnetic field variation, which reverses sign every half of the cable twist pitch. The regions of superconductor, which see a reduction in field, will undergo demagnetization, while the regions, which see a positive field change will almost not change their magnetic moment. This demagnetization will advance in time as the current re-distribution progresses. The drift will saturate when the regions seeing a negative field change have reached total demagnetization. The presence of regions with no demagnetization is presumably also the reason for the fact that the magnets typically undergo only $30-50 \%$ of the total possible demagnetization. The model also naturally explains why the snap-back occurs quickly. As the current ramp resumes, the regions, which saw a decreasing field due to the local current redistribution fields, suddenly experience a positive field change again, which will quickly overcome the redistribution field. The short duration of the snapback is therefore entirely explained by the ramp speed (local $\mathrm{T} / \mathrm{sec}$ ). In the Tevatron dipoles the snapback typically lasts 10 secs, which at a ramp rate from the injection porch of $\sim 1 \mathrm{mT} / \mathrm{sec}$ is consistent with a local demagnetizing field of the order of $10 \mathrm{mT}$, small compared to the injection field (660 mT).

As was pointed out by Haverkamp, the superconducting magnet dynamic effects are in fact 3D effects, because the cable twist-pitch is comparable to the bore size. Cable regions several twist pitches away from the cross-section of interest participate in the dynamic effects. Haverkamp used a model in which the effect of regions away from the cross-section are weighted with a Gaussian function that peaks in the cross-section of interest. Haverkamp also showed via calculation that a random phasing of the twistpitches between the turns, which is the most likely situation in a real magnet, produces the strongest drift signal. This strong averaging of the magnetization response over several twist pitches together with the random phasing are the reasons why the drift and snapback characteristics do not vary strongly along the magnets. A similar, "from-first-principals" model was recently developed by Sugita, [12]. Sugita's calculations indicate that the observed periodic pattern in the KEK LHC-IR quads is consistent with an imbalance current of $\sim 100 \mathrm{~A}$.

\section{MAGNET DATA}

An important aspect of dynamic effects in superconducting accelerator magnets is the magnet-tomagnet variation, which tends to be as large as $100 \%$. This is true for both the Tevatron and the LHC dipole magnets. Possibly it is the result of variations in the inter- 


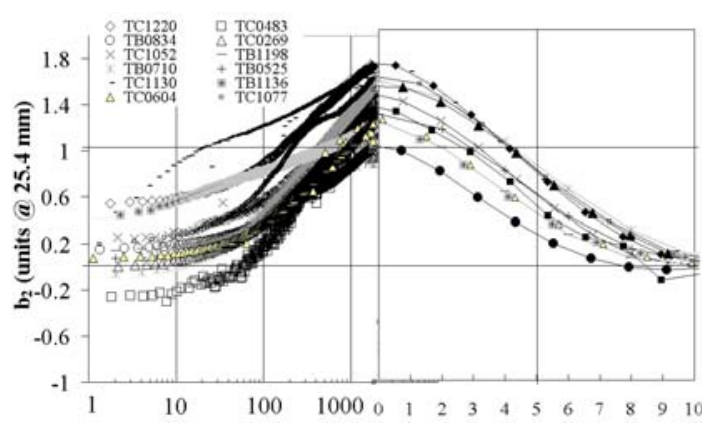

Figure 2: $b_{2}$ drift and snapback (vs time in sec) measured in 12 Tevatron dipoles after a standard pre-cycle at $4.0 \mathrm{~K}$.

strand current dynamics as a result of contact resistance and splice resistance variations. Fig. 2 shows the drift and snapback during and after a 30 min injection porch in a dozen Tevatron magnets measured with a rotating coil system in MTF $[14,18]$. All magnets had been prepared with the same pre-cycle (20 min flat-top at $980 \mathrm{GeV}, 1$ min back-porch at $150 \mathrm{GeV}$, followed by a brief reset at $90 \mathrm{GeV}$ and a ramp to the $150 \mathrm{GeV}$ injection porch). Note that for this plot the underlying geometric and hysteretic $b_{2}$ was removed from the measurement results. Fig. 3 shows similar data for $\sim 50$ LHC dipoles. The standard LHC pre-cycle includes a 30 min flat-top at $8.34 \mathrm{~T}$ (the minimum field reset is at $0.25 \mathrm{~T}$ ).

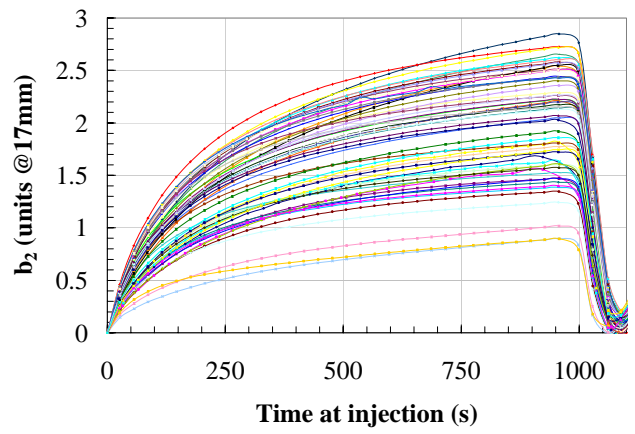

Figure 3: $b_{2}$ drift and snapback measured in 50 LHC dipoles during injection after a standard pre-cycle (1.9 K).

The dynamic effects depend on the ramping history of the magnet because the sequence, duration and sign of the ramps and fixed field dwells strongly influence the amplitude and pattern of the imbalance current. In the recent Tevatron study the main pre-cycle parameters studied were the pre-cycle flat-top and back-porch durations. The back-porch duration was found to be the pre-cycle parameter with the strongest impact on the $b_{2}$ drift behavior. The drift rate decreases with back-porch duration, while it increases with flattop duration. Fig. 4 shows how the flat-top duration effect saturates. The drift amplitude increases with flat-top current, if all other parameters are kept constant. The effects can qualitatively be explained assuming that the imbalance current driven by the up-ramp to the pre-cycle flat-top is more or less cancelled by the down-ramp. The resulting net imbalance current therefore increases as the delay between up and down-ramp increases or when the time between the last

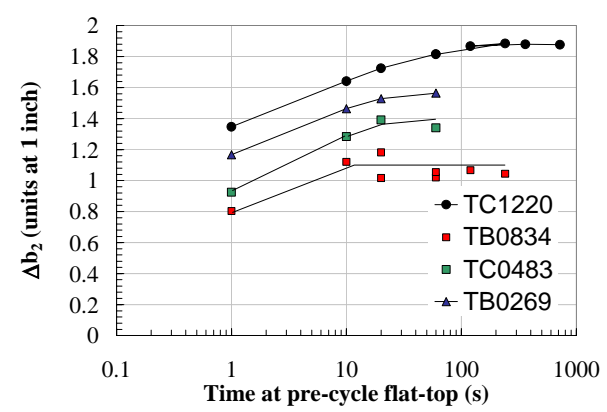

Figure 4: $b_{2}$ drift amplitude after $30 \mathrm{~min}$ at injection for different pre-cycle flat-top durations (1 min back-porch) five Tevatron dipole magnets at $4.0 \mathrm{~K}$.

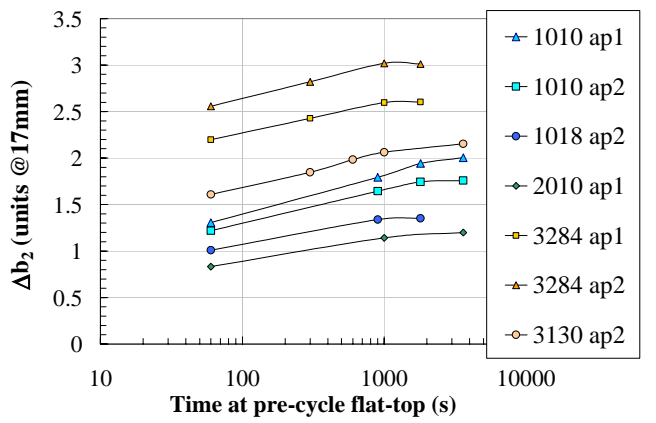

Figure 5: $b_{2}$ drift amplitude after $15 \mathrm{~min}$ at injection for different pre-cycle flat-top durations in several LHC dipole magnets at $1.9 \mathrm{~K}$.

major ramp (down from flat-top) and the injection porch is shortened. Similar observations were made in the case of the LHC. Fig. 5 shows the effect of the flat-top duration on the drift amplitude in several LHC dipoles. The saturation of the drift slope after $\sim 40$ min on the flattop gives a quantitative estimate of the "memory" of the Tevatron magnet. On the basis of a similar argument the memory of the LHC dipoles is $\sim 30$ mins.

The $b_{2}$ drift in Fig. 2 does not always start at zero, but scatters around zero with \pm 0.2 units, which is believed to be some residual of the periodic pattern (and therefore not relevant to the drift). Fig. 2, however, shows that in some magnets the $b_{2}$ drift amplitude departs strongly from zero at $\mathrm{t}=0 \mathrm{sec}$, as if it underwent a very fast drift (and slowed down afterwards). Further exploration of this unexpected, fast drift contribution is under way [21,22]. First measurements were also conducted at CERN to investigate the possibility of fast drifts in the LHC dipoles.

\section{SCALING LAWS}

One of the main results from the recent measurements on LHC and Tevatron dipoles, is that the sextupole and the decapole snap-backs follow a simple exponential law, which applies to all magnets [17,19]. Moreover the fitting parameters of the exponential function, the snap-back amplitude at the beginning of the ramp, $\Delta b_{n}$, and the (magnet) current necessary to resolve it, $\Delta I$, are strongly correlated for all magnets of a given design (Eq. 2). 
$b_{n}^{\text {snap-back }}(t)=\Delta b_{n} e^{-\frac{I(t)-I_{\text {injection }}}{\Delta I}} \Delta b_{n}=\beta_{n} \Delta I$

Fig. 6 shows a summary of the $\Delta b_{2}$ vs. $\Delta I$ parameters extracted from multiple snapback measurements obtained for several different LHC and Tevatron dipoles. The plot also shows that the correlation coefficients are different between LHC and Tevatron magnets. This was shown to be the result of differences in the magnetization transfer function, i.e. the geometrical function that describes how the magnetization response of every strand affects the $b_{n}$ field coefficients in the bore [19]. The scaling law can be applied to a novel, on-line correction approach of sextupole and decapole snap-back in superconducting colliders, such as proposed by Bottura [17]. The idea is to use beam chromaticity measurements at a few times during the injection porch to forecast sextupole and decapole snap-back. The chromaticity measurements can be converted to sextupole change in the dipoles and used to predict the expected sextupole change at snap-back at an arbitrary ramp-time using Eq. 2. The presence of fast drifts, however, could complicate this simple procedure.

\section{REMARKS ON TUNE\&COUPLING DRIFT}

Recent beam studies have revealed that during the injection porch the Tevatron tune drifts by $\sim 0.015$ units over 2 hrs. At the same time the minimum tune split drifts by $\sim 0.02$ units $[15,22]$. The source of this effect remains uncertain to this day. It was first thought that it could be related to drift of the main field component in the arc quadrupoles. Recent measurements of the main field decay in dipoles and quadrupoles [21] revealed that both drifts are approximately of the same order $\left(\Delta b_{0} \sim \Delta b_{1}\right)$. Since only the difference in drift between the relative multipoles can produce changes of the tune, this option had to be dismissed as a leading candidate. Also, the search for possible sources of skew quad drift was not successful [20].

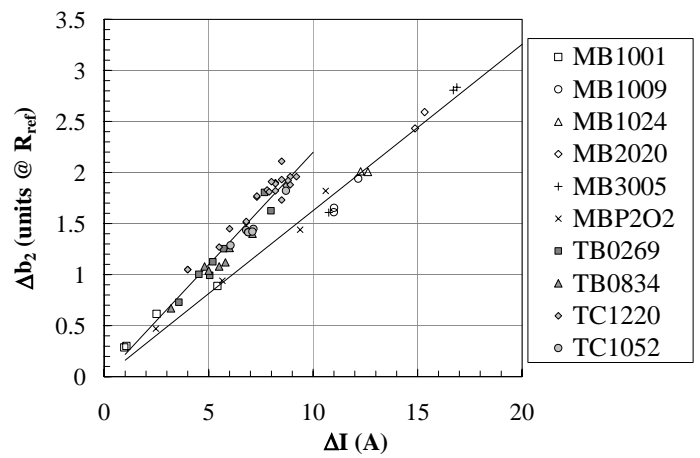

Figure 6: Scatter plot of the fit parameters for the sextupole harmonic $\Delta b_{2}$ and $\Delta I$ in the LHC (MB..) and Tevatron (T..) dipoles.

Several aspects of the tune and coupling drift point toward a connection to the sextupole drift in the dipoles: -
1- the drift is logarithmic, -2- at least in the case of the tune a snapback is observed, -3 - the vertical and horizontal tunes drift by a similar amount but with opposite sign and -4- there are also static tune and coupling components that are not yet explained in the Tevatron (with the dynamic components representing $\sim 10 \%$ of it). A possible mechanism could be feed-down from the sextupole component in the dipoles (and sextupole correctors) as a result of beam offset in the main dipoles and/or sextupole correctors. Systematic beam offset of the order of 0.1-1 $\mathrm{mm}$ in the Tevatron main and corrector magnets suffice to produce the sought amount of feed-down. Measured sagitta errors (giving static and dynamic tune) as well as vertical sag (giving static and dynamic coupling) in Tevatron dipoles are of this order. To this one needs to add yaw and tilt as well as systematic misalignment between the spool-pieces (which contain the sextupole correctors) and the dipoles. An important contributor to the static tune and coupling in this context is the strong $b_{2}$ excursion in the ends of the Tevatron dipoles, which can couple significant $a_{1}$ and $b_{1}$ as a result of feed-down if the beamtrajectory is such that the beam-offset is not the same in body and end, in which case the body-end-compensation in the Tevatron fields is not effective. A large fraction of the recently as being the result of shift of the coils within the magnetic yoke due to creep in the coil suspensions. Harding discusses this issue and its correction in a paper submitted to these proceedings [23].

\section{IMPLICATIONS FOR THE LHC}

The magnet-based measurements indicate that the multipole drifts will be comparable (or even more severe) as those in the Tevatron. The chromaticity change, $\Delta \xi$, for a given amount of $b_{2}$ drift will be stronger in the LHC because the $\xi / b_{2}$ ratio in the LHC is 45 , versus 25 in the Tevatron. The chromaticity is controlled to the level of 1-2 units in the Tevatron. A similar level of control in the LHC will be a challenging task, requiring knowledge of the sextupole in the machine at the 0.05 (magnetic) unit level.

The fraction of LHC dipoles that will be measured at $1.9 \mathrm{~K}$ will most likely not exceed the $20 \%$ mark. While the machine averages of the geometric and hysteretic contributions to the field quality will be well known from warm to cold extrapolations (all magnets will be tested warm), the knowledge of the amplitude of the dynamic effects will be sketchy at best. As is obvious from the example in Fig. 3 the variation between magnets is significant, making extrapolation to the entire magnet population almost impossible. This even more so because the ramping profile used in the drift measurements will most likely not be the one used in the operation. Prediction of the overall drift in the LHC is then further affected by the uncertainty in the pre-cycle parameter correlations (such as in Fig. 5), explored only in a few preseries dipoles.

Overall these issues could make the LHC experience almost as difficult as that of the Tevatron, where the dynamic effects were unknown at start-up (discovered 
after the fact the magnet dynamic effects had to be painfully reconstructed from measurements on spare magnets such as those summarized here). Beam based chromaticity measurements are the only tool available to measure the overall machine average. Such measurements, especially if performed using the variation of the RF frequency, are slow and limited to an accuracy of $\sim 0.1$ magnetic units, close to the operational margin in the LHC. For the above reasons a reference magnet system (RMS) would be a very useful tool during commissioning.

The recently discovered scaling law could simplify the chromaticity drift and snapback correction, because it allows prediction of a feed-forward function for the snapback correction using a measurement of the chromaticity during the injection porch (e.g. with a pilotbunch). Fast drifts, however, as recently discovered in the Tevatron can complicate this on-line correction scheme.

Tune and coupling drifts have to be expected in the LHC as a result of feed-down effects from the sextupole drift in the dipoles and magnet geometrical errors (e.g. sagitta, yaw, tilt).

\section{SUMMARY AND CONCLUSIONS}

Recent extensive magnetic measurement campaigns and beam studies at Fermilab and CERN have generated a large quantity of data on dynamic effects in superconducting magnets. This knowledge has already been used to increase the luminosity in the Tevatron where improved feed-forward compensation schemes were implemented to better correct for the chromaticity, as well as tune and coupling drifts $[13,15,16]$. Fast sextupole drifts were recently discovered at Fermilab, with an understanding still missing. Scaling laws for the snapback $[17,19]$ were developed which have allowed for a simpler and more accurate correction of the chromaticity, tune and coupling snapback. These issues are all of great relevance to the LHC because of its unprecedented beam intensity.

\section{REFERENCES}

[1] D. Finley, D.A. Edwards, R.W. Hanft, R. Johnson, A.D. McInturff, J. Strait, "Time Dependent Chromaticity Changes in the Tevatron”, PAC 12 March 1987, Washington;

[2] R.W. Hanft, B.C. Brown, D. A. Herrup, M. J. Lamm, A.D. McInturff, M.J. Syphers, "Studies of Time Dependence of Fields in Tevatron Superconducting Dipole Magnets”, IEEE Trans. Magn. Vol. 25, No. 2, p. 1647-1651, March 1989 and Fermilab-TM-1542;

[4] M. Kuchnir, A.V.Tollestrup, "Flux Creep in a Tevatron Cable”, IEEE Trans. Magn. Vol. 25, No. 2, March 1989;

[5] L. Krempasky, C. Schmidt, "Theory of "Supercurrents" and their Influence on Field Quality and Stability of Superconducting Magnets", J. Appl. Phys. 78 (9), pp. 5800, 1995;

[6] A.P. Verweij, "Electrodynamics of Superconducting Cables in Accelerator Magnets", PhD thesis, Twente University, Netherlands, 1995;
[7] K.-H. Mess, P. Schmueser, S. Wolff, "Superconducting Accelerator Magnets", World Scientific Publishing, 1996;

[8] L. Bottura, R. Wolf, L. Walkiers, "Field Decay and Snap-back in LHC Model Dipoles", IEEE Trans. Appl. Supercond. Vol. 7(2), pp.602, 1997;

[9] R. Wolf, "The Decay of the Field Integral in Superconducting Accelerator Magnets wound with Rutherford Cables", Proc. of the $15^{\text {th }}$ Int. Magnet Techn. Conf., Beijing, pp. 238, Science Press 1998;

[10] R. Stiening, "A Possible Mechanism for Enhanced Persistent Current Sextupole Decay in SSC Dipoles", SSCL-359, 1991;

[11] M. Haverkamp, "Decay and Snapback in Superconducting Accelerator Magnets”, PhD thesis, Twente University, Netherlands, 2003;

[12] K. Sugita, "Time-Varying Field Quality of Superconducting Accelerator Magnets Induced by Current Redistribution in Cables”, Doctoral Thesis, Tsukuba University, Aug. 2004;

[13] P. Bauer et al., "Analysis of the b2 Correction in the Tevatron”, TPPB055, PAC 2003, Portland, USA, May 2003;

[14] G. Velev et al., "Measurement of Field Decay and Snapback on Tevatron Dipole Magnets", WPAE016, PAC 2003, Portland, USA, May 2003;

[15] M. Martens et al., "Tune and Coupling Drift Compensation in the Tevatron”, FPAB031, PAC 2003, Portland, USA, May 2003;

[16] P. Bauer et al., "Proposals for Improvements of the Correction of Sextupole Dynamic Effects in Tevatron Dipole Magnets”, MOPLT120, EPAC 2004, Lucerne, Switzerland, July 2004;

[17] T. Pieloni et al., "A Scaling Law for Predicting Snapback in Superconducting Accelerator Magnets", WEPKF009, EPAC 2004, Lucerne, Switzerland, July 2004;

[18] G. Velev et al., "Measurement of Decay and Snapback in Tevatron Dipole Magnets", EPAC 2004, Lucerne, Switzerland, July 2004;

[19] L. Bottura et al., "A Scaling Law for the Snapback in Superconducting Accelerator Magnets", presented at the 2004 Applied Superconductivity Conference, Jacksonville USA, and FNAL-Conf-04-230-TD;

[20] P. Bauer et al., "Analysis of Possible Magnet Related Causes of the Tevatron Tune and Coupling Drift and Snapback During Injection”, Fermilab, Technical Division internal note TD-04-052;

[21] G. Velev et al., "Measurements of Field Decay and Snapback Effect on Tevatron Dipole and Quadrupole Magnets”, TPAP029, this conference;

[22] M. Martens et al., "Studies of the Chromaticity, Tune, and Coupling Drift in the Tevatron", MOPA010, this conference;

[23] D.J. Harding et al., "Restoring the Skew Quadrupole Moment in Tevatron Dipoles", MPPT053, this conference; 